\title{
ROSAT Observations of NGC 4258
}

\author{
Andreas Vogler and Wolfgang Pietsch \\ Max-Planck-Institut für extraterrestrische Physik, Giessenbachstr., \\ D-85740 Garching, Germany
}

\begin{abstract}
We performed deep follow up observations of the active spiral galaxy NGC 4258 with the ROSAT HRI and PSPC to study the different emission components. The overall luminosity of NGC 4258 is $L_{\mathbf{x}} \sim 2 \times 10^{40} \mathrm{erg} \mathrm{s}^{-1}$ in the $0.1-$ $2.4 \mathrm{keV}$ ROSAT band. Besides a weak nuclear point source $\left(L_{\mathbf{x}} \lesssim 10^{38} \mathrm{erg} \mathrm{s}^{-1}\right)$ fourteen point sources are detected in the NGC 4258 disk (integral $L_{\mathrm{x}} \sim 3 \times$ $10^{39} \mathrm{erg} \mathrm{s}^{-1}$ ). The bulk of the $\mathrm{X}$-ray emission is not resolved. The main contributions are explained by hot interstellar medium along the anomalous spiral arms of NGC $4258\left(L_{\mathrm{x}} \sim 1 \times 10^{40} \mathrm{erg} \mathrm{s}^{-1}, T \sim 0.4 \mathrm{keV}\right)$ and by interstellar medium escaping from NGC 4258 disk into the approaching halo hemisphere $\left(L_{\mathrm{x}} \sim\right.$ $4 \times 10^{39} \mathrm{erg} \mathrm{s}^{-1}, T \sim 0.2 \mathrm{keV}$ )

Surface brightness, temperature and absorption profiles have been taken for the $\mathrm{X}$-rays in the regions of the anomalous arms. The highest and lowest absorption was received for the NW and SE tips of the arms, respectively. Our X-ray findings are compared to a model of the anomalous arms which suggests that the bar of the galaxy causes shocks in the interstellar medium and might heat a small part of it to X-ray temperatures.
\end{abstract}

\section{The Active Galaxy NGC 4258}

The highly inclined barred spiral galaxy NGC $4258\left(i=72^{\circ}, d=6.4 \mathrm{Mpc}\right)$ hosts a Seyfert 1.9 nucleus (Courtès et al. 1993 and references therein). Courtès \& Cruvellier (1961) discovered a pair of anomalous spiral arms (cf. Fig. 2d). While the arms have been interpreted as twisted jets ejected from the nucleus and tunneling through the disk (cf. Cecil et al. 1995 and references therein), $\mathrm{CO}$ and $\mathrm{H} \alpha$ measurements performed by Cox \& Downes (1996) suggest that the arms are due to interstellar medium excited by bar shocks. The total length of the bar is $\sim 3^{\prime}(5 \mathrm{kpc})$. Water maser emission arising inside the obscuring torus of the AGN has been measured by Miyoshi et al. (1995) indicating the existence of a massive black hole in the center of the galaxy.

We reported on a $7.8 \mathrm{ks}$ observation with the ROSAT PSPC in Pietsch et al. (1994). The low Galactic foreground $\left(N_{\mathrm{H}}=1.2 \times 10^{20} \mathrm{~cm}^{-2}\right)$ allows a sensitive search for X-ray emission in the ROSAT $0.1-2.4 \mathrm{keV}$ band. The emission was separated into three components: Seven point like sources in the spiral arms (total $L_{\mathrm{x}}=2.5 \times 10^{39} \mathrm{erg} \mathrm{s}^{-1}$ ), extended emission from hot $\left(T \sim 4 \times 10^{6} \mathrm{~K}\right)$ interstellar medium above the NGC 4258 disk to the East 
and structured diffuse emission connected to the anomalous spiral arms (altogether $\left.L_{\mathrm{x}}=2.1 \times 10^{40} \mathrm{erg} \mathrm{s}^{-1}\right)$. ROSAT HRI observations $(27 \mathrm{ks}$, Cecil et al. 1995) mostly reveal X-ray emission from the anomalous spiral arms. The authors suggested that the X-rays emerge from hot interstellar medium, which is heated by interactions of the jets with molecular clouds (the jets in this case are believed to be material expelled by the AGN). ASCA revealed hard X-rays from the highly absorbed $\left(N_{\mathrm{H}} \sim 10^{23} \mathrm{~cm}^{-2}\right)$ AGN (Makashima 1994).

\section{Deep ROSAT Follow-Up Observations}

We performed deep follow-up observations of NGC 4258 with the ROSAT PSPC and HRI to better characterize the different emission components. The total PSPC and HRI observation times have been $32.9 \mathrm{ks}$ and $55.8 \mathrm{ks}$, respectively. The (smoothed) images are shown in Fig. 1. Point sources are marked with squares. The central source was detected with a luminosity ${ }^{1}$ of $L_{\mathrm{x}} \sim 1.2 \times 10^{39} \mathrm{erg} \mathrm{s}^{-1}$ The main luminosity of the central source is due to extended emission (cf. Fig 2b). For a possible point source on top of the extended emission we receive $L_{\mathrm{x}} \lesssim 10^{38} \mathrm{erg} \mathrm{s}^{-1}$. This value can be viewed as an upper limit of contributions of the AGN in the ROSAT band. Fourteen point sources besides the X17 are located within the disk of NGC 4258, and they have luminosities in the range $(1-10) \times 10^{38} \mathrm{erg} \mathrm{s}^{-1}$. Three sources (X6, X14, and X18) coincide with HII regions reported by Courtès et al. (1993).

HRI images of the central region of NGC 4258 have been produced using different filtering (Fig. 2a-c). A comparison of the diffuse X-ray emission features with the radio map $(1480 \mathrm{MHz}$; Albada \& van der Hulst 1982; our Fig. 2d) suggests a relationship of the diffuse emission with the anomalous radio arms. In both wavelengths the arms have a position angle of $34^{\circ}$ and bend off at radial distances between 1.5 and $2^{\prime}(3-4 \mathrm{kpc})$ from the nucleus. The HRI images shown in Figs. 2a, $2 \mathrm{~b}$ trace the anomalous arms out to distances of $\sim 2: 7(5 \mathrm{kpc})$ from the center of the galaxy, the radio observations $(2 \mathrm{~d})$ out to $\sim 3.5(6 \mathrm{kpc})$. The adaptively filtered HRI image (2c) might indicate that also the diffuse X-ray emission has such an extent.

Temperature, absorption, and brightness profiles along the anomalous arms ( 9 boxes from the SE tip of the arms to the NW tip) were calculated from the PSPC observations. We measure low and high absorption for the southeast and northwest tips of the anomalous arms, respectively. This might indicate that hot gas along the jets first is confined within the NGC 4258 disk and then escapes into the halo. The gas in the SW flows into the approaching halo hemisphere and is only absorbed by Galactic foreground. A diffuse emission region with a diameter of $r \sim 4^{\prime}-6^{\prime}$ is visible in the PSPC soft (0.1-0.4 keV) band with $L_{\mathrm{x}} \sim 4 \times 10^{39} \mathrm{erg} \mathrm{s}^{-1}$ and $T \sim 0.2 \mathrm{keV}$. The emis-

${ }^{1}$ All luminosities are corrected only for Galactic foreground. 


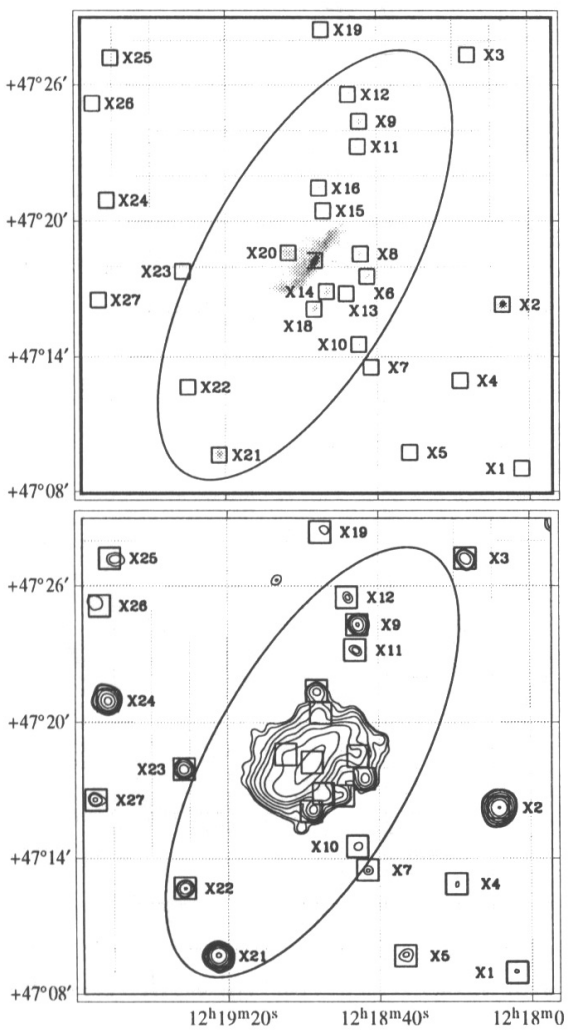

Fig. 1. ROSAT-Observations of NGC 4258 with the HRI (top) and PSPC (bottom). Point sources are marked with squares and enumerated (the central source (X17) has not been enumarated)

sion is displaced from the center towards the SE halo hemisphere and seems to suffer no absorption from cold gas in the NGC 4258 disk. It could be hot interstellar medium ejected from the southwestern arm into the approaching halo hemisphere. The gas in the NW flows into the reverse hemisphere, and the soft X-rays are absorbed by cold gas in the intervening NGC 4258 disk.

Our X-ray findings are compatible with the model of the anomalous arms as suggested by Cox \& Downes (1996). They interpret the anomalous arms as interstellar medium excited by bar shocks. Their $\mathrm{CO}$ observations indicate that the inner parts of the anomalous arms are confined to the galactic disk (there the bar shocks take place), and the gas flows out of the disk in the regions of the radio plateaus at radial distances of $\sim 1.5$ from the nucleus.

We measure an X-ray luminosity from the SE tip to NW tip of the arms of $L_{\mathrm{x}} \sim 9 \times 10^{39} \mathrm{erg} \mathrm{s}^{-1}$ (corrected for Galactic foreground). If we correct for the absorption according to our absorption profiles we obtain an intrinsic 


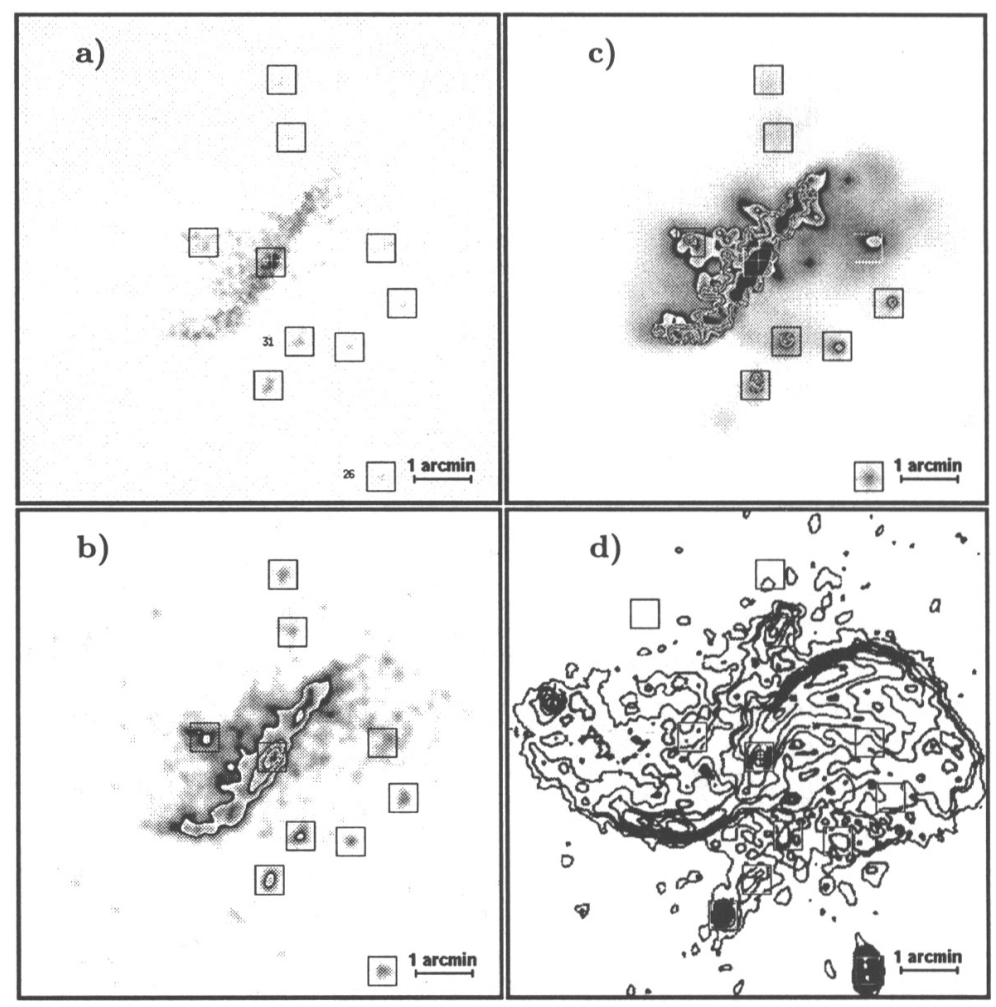

Fig. 2. The inner region of NGC 4258. a) ROSAT HRI image at full resolution (smoothed with a gaussian function of $5^{\prime \prime}$ FWHM) b) at $10^{\prime \prime}$ resolution c) after applying an adaptive filtering technique d) $1480 \mathrm{Mhz}$ radio observation (Albada \& van der Hulst 1982)

luminosity that is higher by a factor of five. The X-ray data allow to estimate the density and mass of the hot gas along the arms. For a filling factor of unity we calculate $n_{\mathrm{e}} \sim 3 \times 10^{-2} \mathrm{~cm}^{-3}$ and $m \sim 7 \times 10^{6} \mathrm{M}_{\odot}$. The values are of the same order of magnitude as predicted by Cox \& Downes (1996).

\section{References}

Albada G.D., van der Hulst J.M., 1982, A\&A 115, 263

Cecil G., Wilson A.S., de Pree C., 1995, Apj 440, 181

Courtès G., Cruvellier P., 1961, Compt.Rend.Acad.Sci.Paris, 253, 218

Courtès G., Petit H., Hua C.T., et al., 1993, A\&A 268, 419

Cox P., Downes D., 1996, ApJ 473, 219

Miyoshi M., Moran J., Herrnstein J., et al., 1995, Nature vol. 373, p. 127

Pietsch W., Vogler A., Kahabka P., et al., 1994, A\&A 284, 386 